Check for updates

Cite this: Chem. Commun., 2020,

56,3879

Received 7th January 2020,

Accepted 25th February 2020

DOI: $10.1039 / \mathrm{d} 0 \mathrm{cc} 00138 \mathrm{~d}$

rsc.li/chemcomm

\section{Photoelectric current as a highly sensitive readout for potentiometric sensors $\dagger$}

\author{
Xu Hun, $\ddagger^{\mathrm{a}}$ Xiaoli Xiong, $\ddagger^{\mathrm{ab}}$ Jiawang Ding $\star^{\mathrm{bcd}}$ and Wei Qin (D) *bcd
}

\begin{abstract}
We report here a general strategy to read out potentiometric signals via a photoelectrochemical method. The photocurrent at a working electrode coated with a ZnSe/r-GO composite can be modulated by a polymeric membrane ion-selective electrode that works as a reference electrode.
\end{abstract}

Potentiometric sensors based on ion-selective electrodes (ISEs) are widely applied in clinical diagnostics, industrial analysis, and environmental monitoring. ${ }^{1,2}$ Their potentiometric responses ideally obey the Nernst equation, which predicts a relatively poor sensitivity for a small ion activity variation. With the introduction of alternative sensing concepts, new ion-selective readout strategies based on chronopotentials, ${ }^{3}$ coulometric charges, ${ }^{4}$ voltammetric currents, ${ }^{5}$ and transition times ${ }^{6}$ have been designed.

In recent years, optical ion sensing platforms have also been reported based on potential-modulated signals. Previous studies by Bakker's group demonstrated electrogenerated chemiluminescence (ECL) to be a promising readout for potentiometric ion sensors. ${ }^{7}$ Both potentiometric and chronopotentiometric signals can be converted to an ECL readout. ${ }^{8,9}$ To simplify the experimental setup, a light emitting diode (LED) was selected as a signal

\footnotetext{
${ }^{a}$ Key Laboratory of Optic-electric Sensing and Analytical Chemistry for Life Science, MOE, Shandong Key Laboratory of Biochemical Analysis, Key Laboratory of Analytical Chemistry for Life Science in Universities of Shandong, College of Chemistry and Molecular Engineering, Qingdao University of Science and Technology, Qingdao 266042, China

${ }^{b}$ CAS Key Laboratory of Coastal Environmental Processes and Ecological Remediati-on, Yantai Institute of Coastal Zone Research (YIC), Chinese Academy of Sciences (CAS), Shandong Key Laboratory of Coastal Environmental Processes, YICCAS, Yantai, Shandong 264003, P. R. China. E-mail: jwding@yic.ac.cn, wqin@yic.ac.cn; Fax: +86535 2109000; Tel: +86 5352109156

${ }^{c}$ Laboratory for Marine Biology and Biotechnology, Pilot National Laboratory for Marine Science and Technology (Qingdao), Shandong 266237, P. R. China

${ }^{d}$ Center for Ocean Mega-Science, Chinese Academy of Sciences, Qingdao, Shandong, 266071, P. R. China

$\dagger$ Electronic supplementary information (ESI) available: Experimental and characterization details, biophysical studies and additional figures as described in the text. See DOI: 10.1039/d0cc00138d

\$ Equal contribution.
}

reporter for field-effect transistors and ISEs. ${ }^{10}$ As an alternative, we designed ion sensing platforms by converting potentiometric signals to a colorimetric readout through the potential-modulated release of enzyme, which could increase the detection sensitivity due to the enzyme amplification effect. ${ }^{11} \mathrm{~A}$ colorimetric readout can also be obtained by the turnover of a redox indicator in a thin layer using a closed bipolar electrode. ${ }^{12}$ Recently, Maksymiuk et al. proposed an approach based on a selffeeding bipolar electrode system for reading the potential response of ISEs with fluorescence. ${ }^{13}$ While these methods are sensitive to and selective for ion detection, new transduction schemes with simple operation, high sensitivity and integrated platforms are still needed.

Photoelectrochemical (PEC) analysis has attracted considerable attention as a newly developed and promising analytical technique. ${ }^{14-16}$ As an evolutionary generation of electrochemistry, the PEC technique is greatly different from the traditional electrochemical methods and offers some merits that could not be realized from the latter. ${ }^{17,18}$ In the PEC method, the photoactive material is excited by a light source and the output signal is in the form of current. The light source and detection signal are completely separated and in different energy forms. Therefore, compared with traditional electrochemical methods, PEC analysis has a higher sensitivity because of its reduced background signals. ${ }^{19,20}$ Also, different from classical potentiometry, amperometric methods can measure the current at the pA level. ${ }^{5}$ Moreover, compared with optical techniques that necessitate complicated and expensive equipment, the utilization of an electronic readout makes the PEC instrumentation simpler, cheaper, and easier to miniaturize. Thus, photoelectrochemical current can be a good option for reading the potentiometric response of ISEs.

Herein, a new strategy is proposed to convert potentiometric signals to PEC readouts. Due to the wide direct band gap $(2.7 \mathrm{eV})$ and its transparency over a wide range of the visible spectrum, zinc selenide ( $\mathrm{ZnSe}$ ) was selected as the photoactive material. Moreover, ZnSe was attached onto reduced graphene oxide (r-GO) to depress electron-hole recombination and 


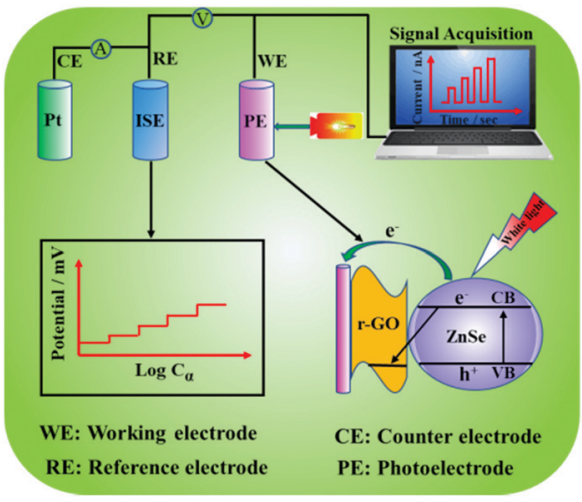

Scheme 1 Schematic diagram of the PEC sensing platform for reading the potentiometric response of an ion-selective electrode.

improve the photocurrent density by making use of the synergistic effect of ZnSe and r-GO. For the PEC technique, the current density generally increases exponentially with a potential change, which works in a similar way to the Schottky diode. ${ }^{21}$ Accordingly, sufficiently large currents are expected when a small potential change occurs on the working electrode during PEC measurements. Since the ISE can be used as the reference electrode to modulate the working electrode potential, ${ }^{7,8,11}$ in principle, a small potential variation can be converted to an obvious change in the photocurrent output. As a proof of concept experiment, a solid-contact ISE for the detection of calcium ions in seawater was prepared and used as a reference electrode for the PEC measurement system.

The PEC sensing platform for reading the potentiometric response of an ISE is shown in Scheme 1. In this setup, a gold electrode (with a diameter of $5 \mathrm{~mm}$ ) modified with a ZnSe/r-GO thin film serves as the working electrode (WE) for producing the photocurrent output. A polymeric membrane ISE is used not only as a potentiometric sensor for ion activities in the sample, but also as a reference electrode (RE) for the sensing platform. During the measurements, a constant potential is applied between the WE and RE, so that the potential of the WE can be modulated by the ISEs. Under visible light illumination, electrons from the valence band (VB) of ZnSe jump to the conduction band (CB) and dissociate into holes $\left(\mathrm{h}^{+}\right)$and free electrons $\left(\mathrm{e}^{-}\right)$, respectively. The transfer of the electrons to the electrode gives rise to a photocurrent. Because of the lower Fermi energy level of r-GO, the direct transfer of photogenerated electrons from the $\mathrm{CB}$ of $\mathrm{ZnSe}$ to r-GO occurs, resulting in a high and stable photocurrent. The photocurrent increases as the working electrode potentials increase and generates more photogenerated electrons. ${ }^{22}$ According to the theoretical model, we derived an expression for the photocurrent (see the ESI $\dagger$ for details). The ion activity changes in the sample solution can be converted to photocurrent $\left(I_{\mathrm{pc}}\right)$ as described as follows:

$$
I_{\mathrm{pc}}=k-k^{\prime} A^{\left[B+k^{\prime \prime} \log \alpha_{x}\right]^{\frac{1}{2}}}
$$

where $k, k^{\prime}, k^{\prime \prime}, A$ and $B$ can be constants.
The ZnSe/r-GO composite was prepared by a facile hydrothermal process. The morphologies of the synthesized composites were characterized by transmission electron microscopy (TEM) images. As shown in Fig. 1A, the particles of ZnSe were wrapped on the surfaces of $\mathrm{r}-\mathrm{GO}$ sheets (The TEM images of the r-GO sheet and ZnSe are shown in Fig. S1A and B, ESI + ). The energy-dispersive spectroscopy (EDS) spectrum (Fig. S1C, ESI $\dagger$ ) shows that the ZnSe/r-GO composite contains only C, Zn and $\mathrm{Se}$, further revealing that $\mathrm{ZnSe}$ is adhered to the surface of r-GO. The X-ray diffraction (XRD) patterns of $\mathrm{ZnSe} / \mathrm{r}-\mathrm{GO}$ were also recorded (Fig. 1B and Fig. S1D, ESI $\dagger$ ). The positions of the diffraction peaks of ZnG-1, 2, 3, 4, and 5 (the composites with the additions of $10,20,30,40$, and $50 \mathrm{mg}$ of GO, denoted as ZnG-1, 2, 3, 4, and 5, respectively) are the same as those of pristine ZnSe without any impurity peaks, indicating that ZnG-1, 2, 3, 4, and 5 were successfully prepared. In addition, the Raman spectrum shows that the characteristic peaks (D and $\mathrm{G}$ bands) of the graphitic material appear at $1339 \mathrm{~cm}^{-1}$ and $1605 \mathrm{~cm}^{-1}$, respectively (Fig. S1E, ESI $\dagger$ ). ${ }^{23}$ The maximum intensity ratio of the D band and G band is 1.52 for ZnG-3, which confirms the efficient reduction of GO (Fig. S1F, ESI $\dagger$ ).

The PEC performance of different electrodes under white light illumination was investigated. As presented in Fig. 1C, $\mathrm{r}-\mathrm{GO} / \mathrm{GE}$ displays a negative photocurrent, which is due to its p-type conductivity. ${ }^{24}$ The introduction of r-GO could effectively suppress electron hole pairs and improve the photo-induced carriers' separation efficiency so as to enhance the photocurrent response. ${ }^{25}$ Indeed, a significantly higher photocurrent could be observed after introduction of r-GO into the ZnSe. To obtain the optimal PEC response, the influences of the content of GO in the $\mathrm{ZnSe} / \mathrm{r}-\mathrm{GO}$ composite, the amount of $\mathrm{ZnSe} /$ $\mathrm{r}-\mathrm{GO}$ and $\mathrm{pH}$ on the photocurrent were investigated. As shown
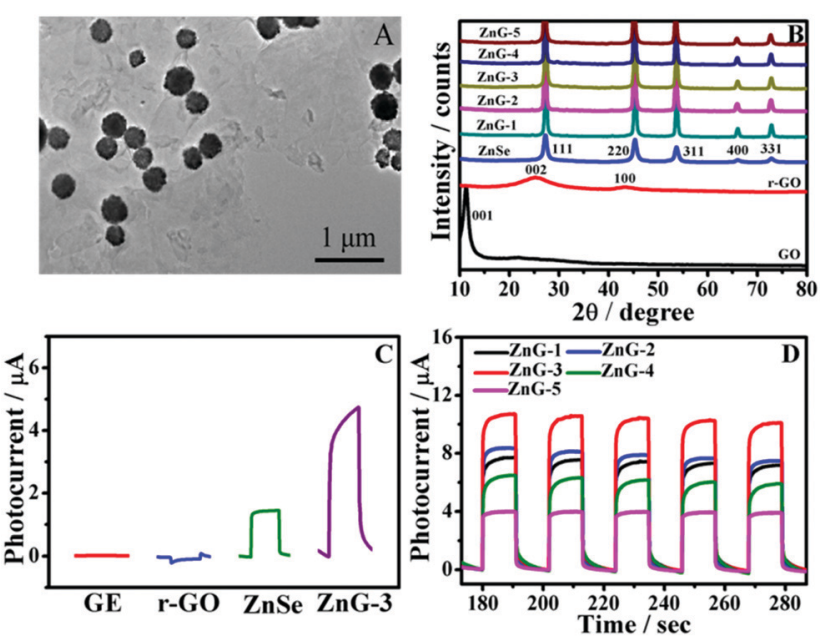

Fig. 1 (A) TEM image of the as-prepared $Z n S e / r-G O$. (B) XRD patterns of the as-prepared GO, r-GO, ZnSe, and the ZnG-1, ZnG-2, ZnG-3, ZnG-4 and ZnG-5 composites. (C) Photocurrent responses of GE, r-GO/GE, $\mathrm{ZnSe} / \mathrm{GE}$ and $\mathrm{ZnG}-3 / \mathrm{GE}$. (D) Time traces of the photocurrent responses of $\mathrm{ZnG}-1,2,3,4$, and 5 composite photoelectrodes at a potential of $0.25 \mathrm{~V}$. The PEC tests were performed using the $\mathrm{Ag} / \mathrm{AgCl}(3 \mathrm{M} \mathrm{KCl})$ electrode in $0.01 \mathrm{M}$ PBS with a $\mathrm{pH}$ of 7.5. A white LED $\left(10 \mathrm{~W}, 10.0 \mathrm{~lm} \mathrm{~W}^{-1}\right)$ light was switched on and off every $10 \mathrm{~s}$. 
in Fig. 1D, the transient photocurrent response increases with an increase in the content of GO in the $\mathrm{ZnSe} / \mathrm{r}-\mathrm{GO}$ composite from $10 \mathrm{mg}$ to $30 \mathrm{mg}$; nevertheless, the PEC response decreases when the content of GO exceeds $30 \mathrm{mg}$. This may be ascribed to the fact that excessive GO in the composites can act as a charge recombination center rather than an electron acceptor thereby leading to the light absorption of $\mathrm{ZnSe}^{26}$ Therefore, the $\mathrm{ZnSe} /$ r-GO composite containing $30 \mathrm{mg}$ of GO was used for the present work. Furthermore, experiments show that the photocurrent response increases significantly along with an increase in the amount of $\mathrm{ZnSe} / \mathrm{r}$-GO coated on the GE surface (Fig. S2A, ESI $\dagger$ ). The maximum photocurrent can be obtained when the amount of the $\mathrm{ZnSe} / \mathrm{r}-\mathrm{GO}$ reaches $0.06 \mathrm{mg}$. Further increasing the ZnSe/r-GO amount can lead to blocking transmission of visible light, thereby decreasing the electron transfer rate and increasing the recombination of the electrons/holes. ${ }^{27}$ Thus, $0.06 \mathrm{mg}$ of $\mathrm{ZnSe} / \mathrm{r}-\mathrm{GO}$ was used for further experiments. Experiments also reveal that the photocurrent versus potential curve is linear for different pHs in the range of 6.0-8.0 (Fig. S2B, ESI $\dagger$ ).

Fig. 2A and B show that the photocurrent responses of ZnG-3 are enhanced on varying the bias potential from -0.20 to $0.50 \mathrm{~V}$, indicating that the photocurrent depends on the working electrode potential. A good linear correlation $\left(R^{2}=0.999\right)$ between the photocurrent and potential can be obtained in the range of $0-0.4 \mathrm{~V}$ (Fig. 2C). More importantly, the current response is sensitive to a rather small potential variation $(<2 \mathrm{mV})$ (Fig. 2D and Fig. S3, ESI $\dagger$ ). Therefore, the photocurrent can be modulated by the potential at the working electrode.

As a proof of concept experiment, the detection of calcium ions in seawater was investigated. A typical $\mathrm{Ca}^{2+}$-ISE that demonstrates a near-Nernstian response of $28.1 \pm 0.9 \mathrm{mV} /$ decade $(n=3)$ with a detection limit of $2.0 \times 10^{-6} \mathrm{M}$ (Fig. S4, ESI $\dagger$ ) was designed. The $\mathrm{Ca}^{2+}$-ISE was used as the reference
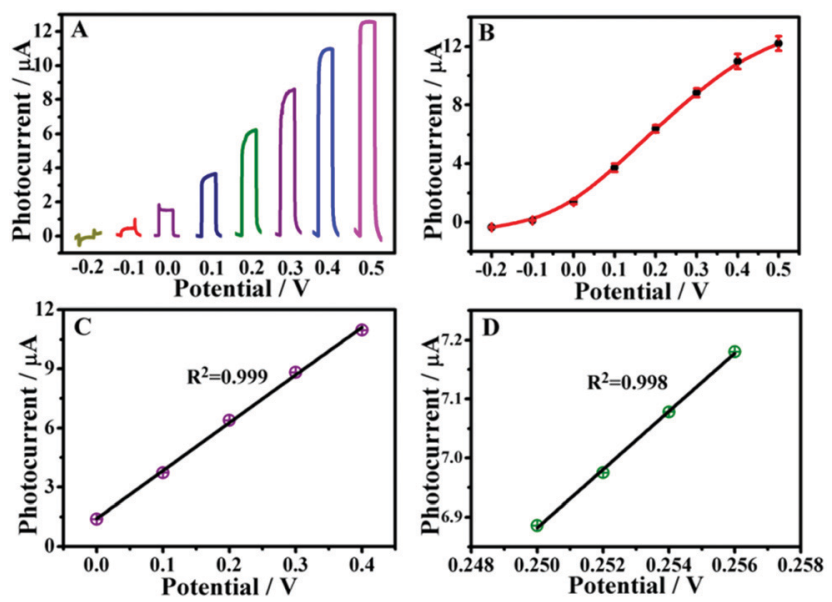

Fig. 2 (A) Photocurrent responses of the $\mathrm{ZnSe} / \mathrm{r}-\mathrm{GO}$ modified GE electrodes with variable potentials from -0.2 to $0.5 \mathrm{~V}$. (B) Plot of the photocurrent vs. potential. (C) Derived calibration curve with potentials from 0 to $0.4 \mathrm{~V}$. (D) Derived calibration curve with potentials from 0.250 to $0.256 \mathrm{~V}$. The PEC tests were performed using the $\mathrm{Ag} / \mathrm{AgCl}(3 \mathrm{M} \mathrm{KCl}$ ) reference electrode in $\mathrm{pH} 7.5 \mathrm{PBS}(0.01 \mathrm{M})$. Each error bar represents one standard deviation of three measurements. electrode in the PEC cell, so that a potential change at the reference electrode alters the resulting working electrode potential, which leads to a change in the photocurrent output. As shown in Fig. S5 (ESI $\dagger$ ), a near-Nernstian response $(-28.3 \mathrm{mV}$ per decade) was observed for the sensor. The slope is negative and the sign of the potential is in the reverse direction, which indicates that the potential at the working electrode of the ZnG-3 composite can be modulated by the potential change at the $\mathrm{Ca}^{2+}$-ISE. Since the potential difference was about $0.15 \mathrm{~V}$ when the $\mathrm{Ag} / \mathrm{AgCl}(3 \mathrm{M} \mathrm{KCl})$ electrode and the $\mathrm{Ca}^{2+}$-ISE $\left(10^{-2} \mathrm{M} \mathrm{Ca}^{2+}\right)$ were used as the reference electrodes, respectively (Fig. S6, ESI $\dagger$ ), $0.25 \mathrm{~V}$ was selected for further experiments. It should be noted that a plasmon-enhanced PEC sensing electrode based on $\mathrm{TiO}_{2} @ \mathrm{Ag} @ \mathrm{Ag}_{3} \mathrm{PO}_{4}$ is available for monitoring $\mathrm{Ca}^{2+} \cdot{ }^{28}$ However, the requirement of the calmodulin functionalization may restrict its wide applications.

It is well known that the change in calcium ion concentration in seawater is rather small. ${ }^{29}$ The responses of the ISE were tested for the activities of calcium varying from $4.2 \times 10^{-3}$ to $6.1 \times 10^{-3}$ M. As can be seen from Fig. $3 \mathrm{~A}$ and B, for potentiometric measurements, a $c a$. $10 \%$ change in $\mathrm{Ca}^{2+}$ activity would correspond to a potential change of only ca. $1.1 \mathrm{mV}$. For $10^{-2} \mathrm{M}$ $\mathrm{Ca}^{2+}$, the relative standard deviation (RSD) was found to be $2.6 \%$. The photoelectrochemical analysis uses the current as the output signal and measurements can be made at the pA level, thus it can offer high sensitivity. Indeed, as shown in Fig. 3C and D, the resulting photocurrent versus activity of calcium is linear in the range of $4.2 \times 10^{-3}-6.1 \times 10^{-3} \mathrm{M}$ and the correlation coefficient $\left(R^{2}\right)$ is 0.997 . A $10 \%$ change in $\mathrm{Ca}^{2+}$
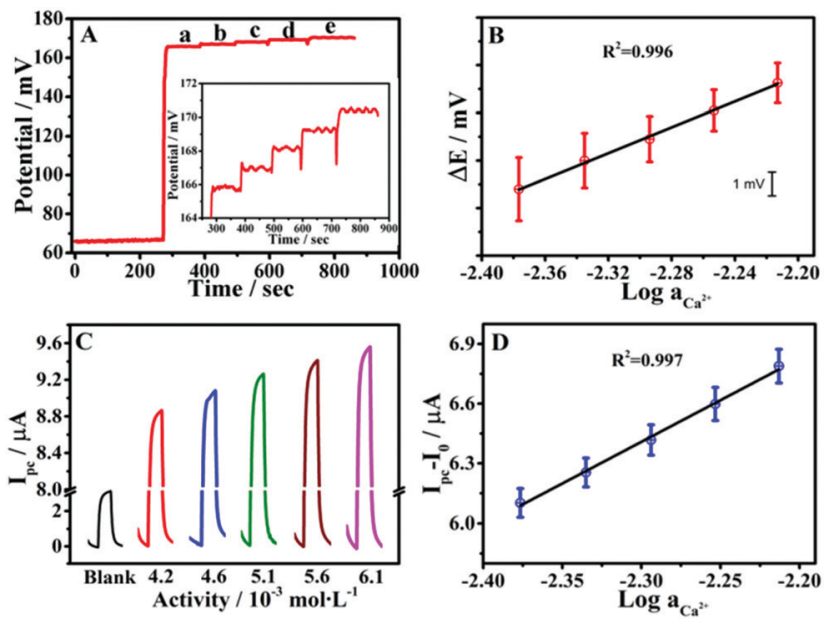

Fig. 3 (A) Time traces of the potential responses of the polymeric membrane $\mathrm{Ca}^{2+}-$ ISEs for detecting various activities of $\mathrm{Ca}^{2+}$ in $10^{-2} \mathrm{M}$ $\mathrm{NaCl}$ : (a) $4.2 \times 10^{-3}$, (b) $4.6 \times 10^{-3}$, (c) $5.1 \times 10^{-3}$, (d) $5.6 \times 10^{-3}$, and (e) $6.1 \times 10^{-3}$ M. (B) Corresponding calibration curve. (C) Photocurrent responses of the $\mathrm{ZnSe} / \mathrm{r}$-GO modified GE electrodes with variable calcium activities from $4.2 \times 10^{-3}$ to $6.1 \times 10^{-3} \mathrm{M}$. (D) Corresponding calibration curve. $I_{0}$ is the photocurrent intensity measured in a $10^{-2} \mathrm{M} \mathrm{NaCl}$ background solution without calcium, and $I_{\mathrm{pc}}$ is the intensity after the addition of calcium. The PEC tests were performed with a $0.25 \mathrm{~V}$ applied voltage (vs. $\mathrm{Ca}^{2+}-\mathrm{ISE}$ ). Each error bar represents one standard deviation of three measurements. 
Table 1 Results of determination of calcium in seawater samples ${ }^{a}$

\begin{tabular}{llll}
\hline \multicolumn{2}{l}{ Samples ICP-OES $\left(\mathrm{g} \mathrm{L}^{-1}\right)$} & Conventional Ca-ISE $\left(\mathrm{g} \mathrm{L}^{-1}\right)$ & This work $\left(\mathrm{g} \mathrm{L}^{-1}\right)$ \\
\hline Site 1 & $0.450 \pm 0.012$ & $0.448 \pm 0.021$ & $0.452 \pm 0.010$ \\
Site 2 & $0.454 \pm 0.011$ & $0.452 \pm 0.016$ & $0.457 \pm 0.009$ \\
Site 3 & $0.442 \pm 0.008$ & $0.431 \pm 0.023$ & $0.449 \pm 0.002$ \\
\multicolumn{2}{l}{ a } &
\end{tabular}

activity was detectable and the photocurrent difference was up to hundreds of $\mathrm{nA}$, indicating that the proposed method can remarkably improve the sensitivity for $\mathrm{Ca}^{2+}$ ion detection. Compared with the potentiometric method, the RSD was $1.2 \%$ for the detection of $10^{-2} \mathrm{M} \mathrm{Ca}^{2+}$ by using the photoelectrochemical method. Therefore, this proposed method exhibits a high resolution for the detection of calcium in seawater. Moreover, the PEC sensor demonstrates acceptable stability and reproducibility for the detection of $\mathrm{Ca}^{2+}$ (Fig. S7, ESI $\dagger$ ). It should be noted that the electrolyte environment variations may exert effects on the physical and chemical processes involved in the generation of the electrical signal that triggers the PEC signal alterations (as indicated in Fig. 2 and 3). ${ }^{15}$

In order to explain the rationality of the results, a theoretical simulation of photoelectrochemical response was performed for detecting $\mathrm{Ca}^{2+}$ ions. As shown in Fig. S8 (ESI $\dagger$ ), the theoretical simulation (parameters used for the simulation are given in Table S1, ESI $\dagger$ ) was almost identical to the experimental data, indicating that the result was reasonable.

The average concentrations as well as the standard deviations of calcium measurements in real seawater were measured by using the proposed method, and conventional potentiometric ISE and ICP-OES methods (see Table 1). Experiments reveal that no obvious photocurrent change can be observed in the presence of high concentrations of $\mathrm{NaCl}$ (0.5 M, Fig. S9, ESI $\dagger$ ). For the photoelectric and potentiometric measurements, the standard addition method was used to detect the amount of $\mathrm{Ca}^{2+}$ ions in seawater. Table 1 shows that the results obtained by the proposed photoelectrochemical sensor agree well with those of the other two reference methods. More importantly, the proposed method has a high resolution compared with the traditional potentiometric ISE.

In summary, a general approach to read out potentiometric signals by a photoelectrochemical method is proposed. This proposed technique offers several advantages over other methods. Firstly, in contrast to the conventional Ca-ISE with the Nernstian response, the present sensor exhibits high sensitivity and can be used for detection of small variations in ion activity (e.g., electrolyte measurements in clinical diagnosis). Secondly, the PEC technique is simple, integrated and easy to miniaturize. Thirdly, by changing the ion-selective electrode that serves as a reference electrode, the proposed method is promising for detecting a variety of different ions with high sensitivity.

This work was supported by the National Natural Science Foundation of China (41876108, 21575158, 21575073, and 21677172), the Laoshan Scholar Program of Qingdao University of Science and Technology (201802685), The Instrument Developing Project of the Chinese Academy of Sciences (Y728021021), the National Key Research and Development Program of China (No. 2016YFC1400700), the Taishan Scholar Program of Shandong Province (tspd20181215, tsqn201909-163) and the Key Research and Development Program of Yantai (2018ZHGY053).

\section{Conflicts of interest}

There are no conflicts to declare.

\section{Notes and references}

1 E. Bakker, P. Bühlmann and E. Pretsch, Chem. Rev., 1997, 97, 3083-3132.

2 J. Bobacka, A. Ivaska and A. Lewenstam, Chem. Rev., 2008, 108, 329-351.

3 A. Shvarev and E. Bakker, J. Am. Chem. Soc., 2003, 125, 11192-11193.

4 E. Bakker, Anal. Chem., 2011, 83, 486-493.

5 X. Nagy and L. Höfler, Anal. Chem., 2016, 88, 9850-9855.

6 G. A. Crespo, M. G. Afshar and E. Bakker, Angew. Chem., Int. Ed., 2012, 51, 12575-12578.

7 G. A. Crespo and E. Bakker, Analyst, 2012, 137, 4988-4994.

8 G. A. Crespo, G. Mistlberger and E. Bakker, J. Am. Chem. Soc., 2012, 134, 205-207.

9 W. Y. Gao, S. Jeanneret, D. J. Yuan, T. Cherubini, L. Wang, X. J. Xie and E. Bakker, Anal. Chem., 2019, 91, 4889-4895.

10 X. W. Zhang, Y. C. Han, J. Li, L. B. Zhang, X. F. Jia and E. K. Wang, Anal. Chem., 2014, 86, 1380-1384.

11 J. W. Ding, E. G. Lv, L. Y. Zhu and W. Qin, Anal. Chem., 2017, 89, 3235-3239.

12 S. Jansod, M. Cuartero, T. Cherubini and E. Bakker, Anal. Chem., 2018, 90, 6376-6379.

13 E. Jaworska, A. Michalska and K. Maksymiuk, Electrochim. Acta, 2018, 284, 321-327.

14 L. Y. Xia, M. J. Li, H. J. Wang, R. Yuan and Y. Q. Chai, Chem. Commun., 2019, 55, 9721-9724.

15 J. Shu and D. P. Tang, Anal. Chem., 2020, 92, 363-377.

16 Y. Zhang, N. Hao, Z. Zhou, R. Hua, J. Qin, Q. Liu, H. N. Li and K. Wang, Chem. Commun., 2017, 53, 5810-5813.

17 W. W. Zhao, J. J. Xu and H. Y. Chen, Chem. Soc. Rev., 2015, 44, 729-741.

18 W. W. Zhan, Q. Kuang, J. Z. Zhou, X. J. Kong, Z. X. Xie and L. S. Zheng, J. Am. Chem. Soc., 2013, 135, 1926-1933.

19 H. M. Da, Y. L. Liu, M. J. Li, R. Yuan, H. Y. Liu and Y. Q. Chai, Chem. Commun., 2019, 55, 8076-8078.

20 M. M. Liang, S. L. Liu, M. Y. Wei and L. H. Guo, Anal. Chem., 2006, 78, 621-623.

21 P. P. Hankare, P. A. Chate, D. J. Chate, M. R. Asabe and B. V. Jadhav, Solid State Sci., 2008, 10, 1970-1975.

22 K. Rajeshwar, Encycl. Electrochem., 2007, 6, 1-562.

23 H. H. Li, J. Yang, C. L. Liang, W. Zhang and M. Zhou, Appl. Surf. Sci., 2012, 258, 8959-8964.

24 H. M. El-Bery, Y. Matsushita and A. Abdel-moneim, Appl. Surf. Sci., 2017, 423, 185-196.

25 D. Wang, N. Gan, H. R. Zhang, T. H. Li, L. Qiao, Y. T. Cao, X. R. Su and S. Jiang, Biosens. Bioelectron., 2015, 65, 78-82.

26 R. Z. Li, Y. Liu, L. Cheng, C. Z. Yang and J. D. Zhang, Anal. Chem., 2014, 86, 9372-9375.

27 K. Yan, Y. Liu, Y. H. Yang and J. D. Zhang, Anal. Chem., 2015, 87, 12215-12220.

28 J. Tang, J. Li, D. Sikdar, B. Kong, Y. Z. Quan, S. Che, Y. Wang, A. M. Al-Enizi, M. Premaratne and G. F. Zheng, J. Electroanal. Chem., 2015, 759, 14-20.

29 S. W. Liu, J. W. Ding and W. Qin, Anal. Chim. Acta, 2018, 1031, 67-74. 